\title{
EDITORIAL
}

\section{SAPS AND TEARS}

No disease is too rare for the patient afflicted by it or a condition too minor for the person who suffers it and, one might add, for the doctor who has to treat it. The set thought processes of a routine outpatient clinic are, every now and again, jolted by the patient who walks in with a funny-looking eye that makes the treating surgeon want to disappear into the back rooms to consult a book or journal.

Two reports in this issue that describe ocular injury and inflammation caused by toxic plant sap, illustrate such a situation. Both these reports serve to rekindle our awareness of the exotic and unusual. Many plants, not all of which are 'weeds', can cause severe ocular surface injury and inflammation with associated hypopyon uveitis via their sap, hairs or sharp ends, be it the point of a thorn or the razor edge of a leaf. Clinical features include hyperaemia, lacrimation, follicular reaction, chemosis, corneal infiltrates, punctate keratitis and erosions, epithelial thinning and loss, stromal oedema and infiltration and iridocyclitis with frank hypopyon. A mild to severe keratoconjunctivitis is the hallmark.

Plants of the family Euphorbiaceae and genus Rhus produce a particularly virulent toxic sap and are notorious in this regard. Other examples are Craetegus oxycantha (whitethorn); spores of mushrooms; saps of tropical wood plants such as teak, ebony and satin wood; latex of Semecarpus atra and Anacardiaceae (mango), leaves of Primula abconica (primula); pollens of pigweed and red root; flowers of Pyrethrum carneum and Pyrethrum roseum, and the dried root of the Brazilian Cephoelis ipecacuanha. The active (toxic) agents in plant saps include oxalic, helvellic and chelinonic acids; diterpene esters; volatile oils (turpentine); resins (podophyllum); saponins; phenolic compounds such as urushiol; alkaloids such as cephaeline and emetine; protein substances such as ricin; and other substances such as capsicum and elaterin. At times the ocular manifestations are a result of allergy, particularly following repeated contact with plant products.

When dealing with apparent plant injuries one must not forget to consider the possibility of ocular insult by insects or their larvae that may have been inadvertently disturbed. Moth caterpillar hairs and hairs of other insects and spiders can penetrate the conjunctiva to cause granulomatous conjunctivitis (ophthalmia nodosa), the cornea to cause leucocytic cuffing, and may even access the anterior chamber with ensuing iridocyclitis. Insect stings can cause severe stromal keratitis and uveitis by toxic (mellitin) or allergic (phospholipase A) agents. Beetles that fly into the conjunctival sac may be accidently crushed by eye rubbing to release toxic body fluids (cantharidin) causing keratoconjunctivitis.

Management principles are similar for toxic plant and animal injuries: copious irrigation, examination under topical anaesthesia with double eversion of the upper lid to identify and remove vegetable matter and insect body parts, topical steroids and iridocycloplegia if inflammation is severe. Topical antibiotics may be used prophylactically. Plant and animal hairs should be removed where possible. These are not easy to visualise and are often better felt by drawing the tip of a forceps across the surface. A faint 'click' is felt when the forceps contacts the end of a protruding hair. Conjunctival granulomata may have to be excised together with the encased hair. Hairs that are buried deep in the corneal stroma may be observed and an attempt at removal should be made only if inflammation is persistent. In most cases the prognosis is good.

H. S. DuA

\section{References}

1. Wihelmus KR. Parasitic keratitis and conjunctivitis. In: Smolin G, Thoft RA, editors. The cornea, 3rd ed. Boston: Little Brown, 1994: 262-75.

2. Duke Elder S. System of ophthalmology, vol XIV, part II, Non-mechanical injuries. London: Henry Kimpton, 1972:1180-207. 